\title{
1 DEVELOPMENT OF A BIODEGRADABLE SENSOR PLATFORM FROM GOLD \\ 2 COATED ZEIN NANOPHOTONIC FILMS TO DETECT PEANUT ALLERGEN, ARA \\ 3 H1, USING SURFACE ENHANCED RAMAN SPECTROSCOPY \\ P. GIZEM GEZER ${ }^{\mathrm{a}}$, G. LOGAN LIU ${ }^{\mathrm{b}, \mathrm{c}}$, JOZEF L. KOKINI ${ }^{\mathrm{c}, \mathrm{d} *}$ \\ $8{ }^{a}$ Department of Food Science and Human Nutrition, University of Illinois Urbana-Champaign, \\ 91304 W. Pennsylvania Ave, Urbana, USA
}

$11{ }^{\mathrm{b}}$ Department of Electrical and Computer Engineering, University of Illinois at Urbana-

12 Champaign, 1406 W. Green St, Urbana, USA

$14{ }^{\mathrm{c}}$ Micro and Nanotechnology Laboratory, University of Illinois at Urbana-Champaign, 13208

15 North Wright Street, Urbana, USA

${ }^{\mathrm{d}}$ Department of Food Science, Purdue University, 745 Agricultural Mall drive, West 7 Lafayette

18 Indiana, 47907

*Corresponding Author: JOZEF L. KOKINI, jkokini@ @urdue.edu Department of Food

21 Science, Purdue 19 University, 745 Agricultural Mall drive, West Lafayette Indiana, 47907 
Peanuts are among the most common food allergies, which may result in life-threatening reactions in certain people. For this reason, it is very important to monitor the presence of

36 peanuts in the food system. Biosensors are an emerging way of detecting allergen proteins. In

37 this research, we present a surface enhanced Raman spectroscopy (SERS) technique to detect the 38 main allergen protein, Ara h1. The sensors were biodegradable and made out of a corn protein, 39 zein. Nanophotonic structures on zein films consisted of gold coated pyramid structures. It was

40 found that both detection and quantification was possible by using a statistical clustering 41 technique principal component analysis (PCA). An optimization in data processing yielded the

42 result that baseline correction and shorter data collection times were needed in order to 43 successfully cluster data. The limit of detection was found to be $0.14 \mathrm{mg} / \mathrm{ml}$. Furthermore, 44 specificity of the sensor was provided by functionalizing the surface with monoclonal antibodies 45 of Ara h1. Antibody functionalization, and Ara h1 capturing was tested and identified by also 46 utilizing PCA analysis. As a proof-of-concept, this study showed that a biodegradable platform 47 can be used in detection of a peanut allergen protein, Ara h1, using surface enhanced Raman 48 spectroscopy.

58 Keywords: Raman, SERS, allergen, zein, Ara h1 


\section{Introduction}

Peanuts are one of the leading causes of food allergy, along with tree nuts, cow's milk, eggs, fish, seafood, soy, wheat and additives [1]. The reaction to peanuts ranges from mild oral effects to potentially fatal ones such as anaphylaxis, which results in itchy rash, low blood pressure and throat swelling. Currently, there is no known antidote for peanut allergy. Prevention relies solely on avoiding peanuts and peanut containing food products [2]. For this reason, it is very important

72 for the food industry to monitor the presence and cross-contamination of peanut proteins in

73 various food products. The most commonly used monitoring technique is based on enzyme-

74 linked immunosorbent assays (ELISA), by either using monoclonal or polyclonal antibodies [3].

75 These methods target the detection of the allergenic proteins of peanuts, which are identified as

76 Ara h1 - h8. Among these, Ara h1 constitutes the largest portion of allergenic proteins in

77 peanuts. In addition, it causes various degrees of allergenic reactions in more than $95 \%$ of the susceptible population. [4-5]. Detection systems as a result, target mostly the Ara h1 protein. Besides detecting the protein itself, there are several studies that detect the DNA responsible for the production of Ara h1 protein. Polymerase chain reaction (PCR) with gel electrophoresis, realtime PCR and PCR-ELISA are some of the techniques that have been utilized to detect the DNA of Ara h1 [6].

83 Recently, surface enhanced Raman spectroscopy (SERS) was utilized to detect the DNA of 84 peanuts, by tagging the DNA with dyes [2]. Raman spectroscopy is a strong molecular

85 fingerprinting technique; however it relies on an inherently weak scattering phenomenon.

86 Surface Enhanced Raman Spectroscopy has proven to be a promising technique, which enhances

87 the intensity of the Raman signal and makes it useful for many sensor applications. The majority

88 of the SERS applications were for small molecules in pure systems, an example being drug

89 applications. Working with a food system, which is more complex, poses its own challenges to

90 the biosensor development. There have been recent developments in utilizing SERS as a

91 potential detection tool for several contaminants in food, such as pesticides, melamine and

92 pathogens [7-9] This paper will discuss a method that was developed to detect the Ara h1

93 protein with SERS. The platform for the sensor was based on a biodegradable corn protein that is 
94 coated with gold nanopatterns for the enhancement of Raman signal. This is a very novel 95 approach and was attempted for the first time. Principal component analysis was employed for 96 both detection and quantification purposes.

2. Materials and Methods

\subsection{Materials used}

Natural Ara h1 and its monoclonal antibody, 2F7 Anti Ara h1 were purchased from Indoor

103 Biotechnologies (Charlottesville, VA). In order to avoid freeze-thaw cycles, both allergen protein 104 and antibody was divided into 5 or $10 \mu \mathrm{l}$ aliquots and stored at $-20^{\circ} \mathrm{C}$. The required number of 105 aliquots were thawed at room temperature before direct use. The concentrations used for Ara h1 106 were $1.4 \mathrm{mg} / \mathrm{ml}, 0.5 \mathrm{mg} / \mathrm{ml}, 0.25 \mathrm{mg} / \mathrm{ml}$ and $0.14 \mathrm{mg} / \mathrm{ml}$. 11-Mercaptoundecanoic acid (11107 MUA), $N$-(3-Dimethylaminopropyl)- $N$ '-ethylcarbodiimide hydrochloride (EDC) and ( $N$ 108 Hydroxysuccinimide) NHS needed to crosslink the 2F7 Anti Ara h1 onto the zein/gold SERS 109 platform were purchased from Sigma Aldrich. 11-MUA and NHS were stored in a refrigerator, 110 whereas EDC was stored in the freezer section of the lab refrigerator at $-20^{\circ} \mathrm{C}$. The solutions 111 were freshly prepared before each use. 11-MUA solutions were prepared in 100\% ethanol. For 112 all the other solutions or dilutions, MilliQ purified water was used.

\section{$114 \quad$ 2.2.Preparation of SERS-active substrates}

115 SERS-active zein substrates were fabricated as described previously [10]. Polydimethylsiloxane 116 (PDMS) with positive pyramid structures were used as a template. The template had pyramids 117 with the base squared dimensions of $2 \mu \mathrm{m}$ by $2 \mu \mathrm{m}$ and a height of $2.1 \mu \mathrm{m}$. The PDMS template 118 was coated with $200 \mathrm{~nm}$ gold by using e-beam evaporator. Zein solution was prepared by first 119 dissolving zein in $75 \%$ ethanol (1g: $5 \mathrm{~mL}$ ) and mixing with oleic acid and emulsifier (1g zein: 120 1g oleic acid: $0.05 \mathrm{~g}$ emulsifier). This solution was stirred on a hot plate for 5 minutes. After 121 ultrasonification to remove air bubbles, zein solution was cast on PDMS template with gold 122 coating. The solution cast on PDMS was allowed to dry and form a film and after drying in a 123 vacuum desiccator at room temperature, dried zein films were cut and peeled off from the PDMS 
124 base. $200 \mathrm{~nm}$ gold along with the pyramid structures were transferred onto zein, resulting in an

125 inverted pyramid structure on zein.

\subsection{Functionalization of zein-SERS substrates}

129 The gold surface of zein-SERS substrates was functionalized with the monoclonal antibody of

130 Ara h1, 2F7. The functionalization scheme is illustrated in Figure 1. First, the surface was

131 functionalized with 11-MUA ethanolic solution (2 M) by dropping $50 \mu 1$ of solution on the gold

132 surface of the SERS platform. The 11-MUA functionalized gold/zein platform was kept in a

133 humidity-controlled incubator for 16 hours. Then, the surface was rinsed both with ethanol and

134 water, followed by drying with a nitrogen stream. Second, carboxylic groups of 11-MUA were

135 activated using the EDC: NHS solution. A 1:1 mixture of 0.4 M EDC and 0.1 M NHS was

136 applied to the gold surface by dropping $50 \mu \mathrm{l}$ of solution and incubating for 45 minutes. The

137 same washing procedure was applied as before. Third, $30 \mu \mathrm{l}$ of anti-Ara h1 with the

138 concentration of $50 \mu \mathrm{g} / \mathrm{ml}$ was dropped on top of the EDC-NHS activated surface. It was

139 incubated for 30 minutes, before washing out the excess using the same procedure.

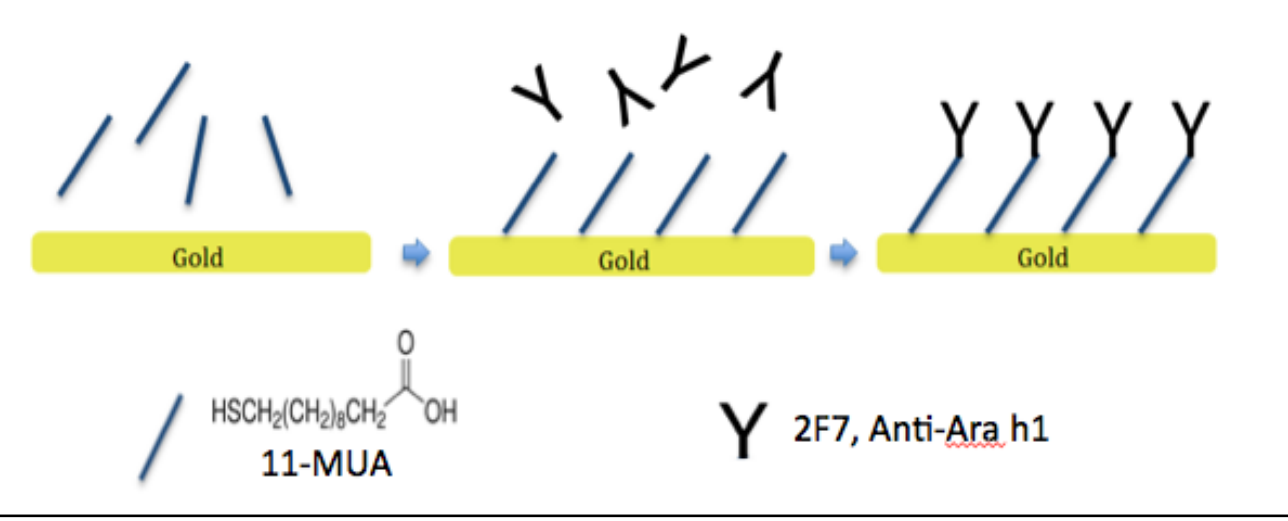

Figure 1. Schematic illustration of the functionalization of the gold surface

\section{2.4.Raman spectroscopy measurements}

145 Samples for Raman spectroscopy were prepared by placing $2 \mu 1$ of Ara h1 solution at one of the 146 concentrations used in this study on one of the two platforms (either smooth gold surface or zein-

147 SERS sensor) tested in this study and letting it air dry at room temperature. The zein/gold 
148 functionalized surface was immobilized onto a glass slide in order to obtain a flat and stable

149 surface. Background Raman spectra were obtained on the nanophotonic structures that did not

150 have any Ara h1 protein solution for comparison with those that had the protein solution. The

151 HORIBA LabRAM HR Raman Spectroscope was used with a $532 \mathrm{~nm}$ laser. The hole diameter

152 was $300 \mu \mathrm{m}$, the slit selected was $100 \mu \mathrm{m}$ and the grating used was 300 lines $/ \mathrm{mm}$. The Raman

153 spectrum was recorded in the wavenumber range between 500 and $1800 \mathrm{~cm}^{-1}$. Spectra were

154 collected 30 times for a period of 1 second of 5 times for a period of 1 second. Each individual

155 final spectrum used is an average of these acquisitions. X-Y Mapping was used to collect data on

156 a $30 \mu \mathrm{m}$ by $30 \mu \mathrm{m}$ square. For the functionalized samples, after Ara h1 solution application with

157 the concentration of $0.2 \mathrm{mg} / \mathrm{ml}$, the surface of the SERS film was dried and the surface was

158 washed with ethanol and water and dried again.

\subsection{Data Analysis}

161

162 For each Raman spectrum, a baseline correction was carried out in order to eliminate the effect

163 of fluorescence interference. A polynomial was fit to the baseline with the 'baseline correction'

164 option of the software LabSpec 5 (provided by Horiba). Additional points were added to the

165 baseline to ensure a perfect fit, if needed. This baseline was subtracted from the Raman

166 spectrum. Principal Component Analysis (PCA) was done using Matlab R2013a software

167 Different Principal Components (PCs) were plotted and color-coded by using the same software.

\section{Results and Discussion}

\subsection{Detection of Ara h1 on zein-SERS substrates}

\section{Analysis of raw data}

173 Raman spectra using zein-SERS substrate give a background signature. For this reason, the first

174 step in this study was to identify the characteristic Raman peaks resulting from the allergen

175 protein, Ara h1. However, since both Ara h1 and underlying zein are proteins, all of the peaks

176 overlapped. This resulted in a challenge in detection and quantification of Ara h1 through our

177 zein-gold platform. A statistical clustering technique, Principal Component Analysis (PCA) was 
178 utilized to discriminate various samples from each other [11-12]. In this study, the PCA

179 technique was used to obtain characteristic clusters of the raw Raman spectra data, baseline

180 corrected data at different acquisition times as well as different concentration of the allergen

181 protein.

182 For each sensor, Raman spectra were collected by mapping measurements at 9 separate

183 points. Example spectra are shown in Figure 2. Raman spectra in Figure 2.a and b were collected

184 by using 30 separate measurements at each point for 1 second, and the Raman spectra in Figures

185 2.c and d were collected by using 5 separate measurements at each point for 1 second. Figures

186 2.a and c show the background signal coming from the zein-SERS sensor, whereas Figures 2.b

187 and c represent the spectra when Ara h1 was deposited on the zein-SERS sensor. As evident

188 from these figures, there was different degrees of significant auto fluorescence in the Raman 189 signal.
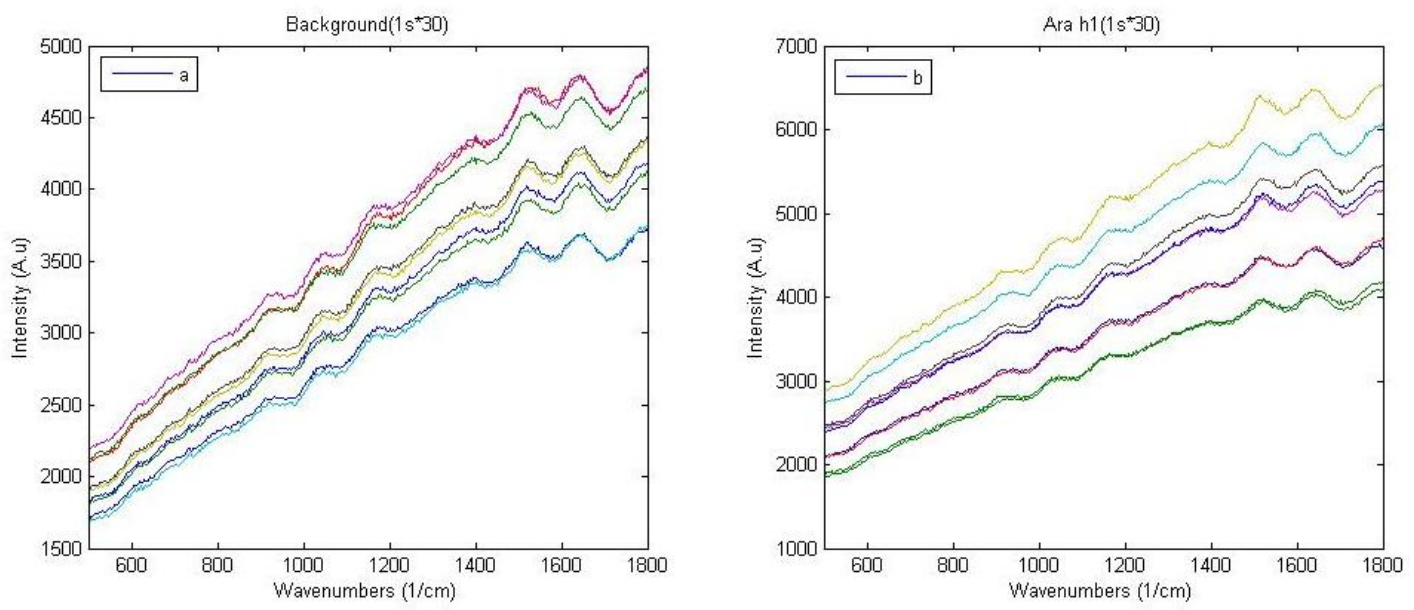

190
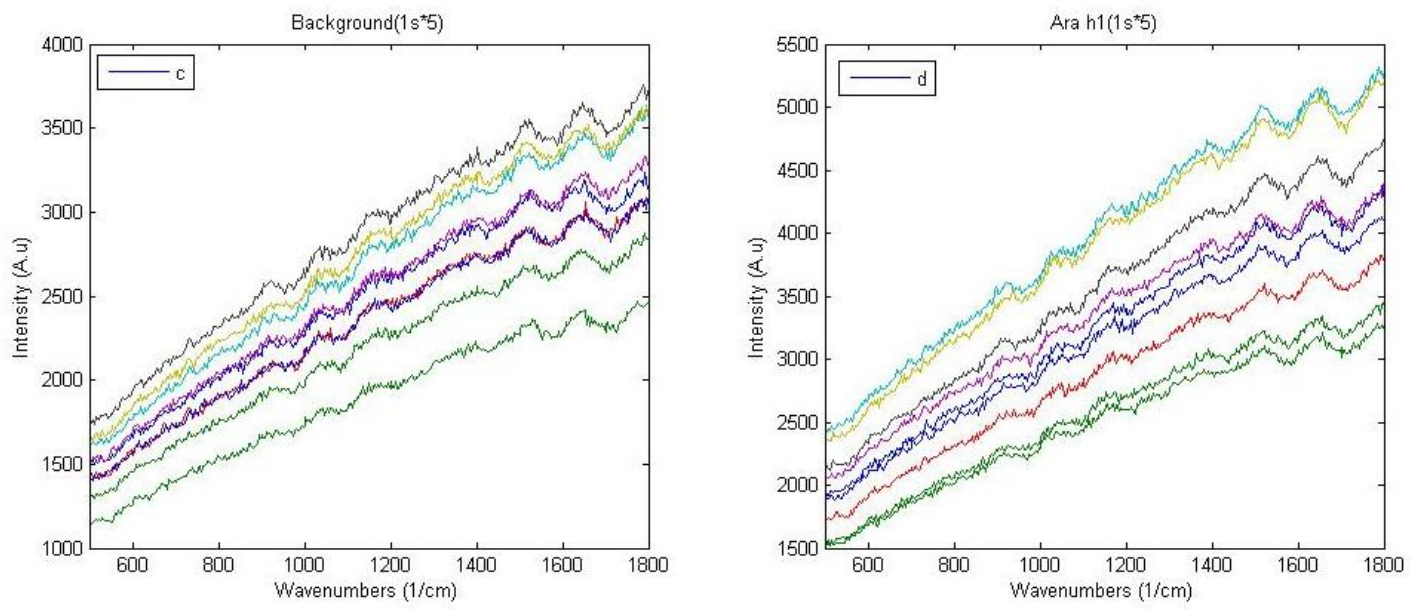
191 Figure 2. Raman spectra of a) background of the zein-SERS sensor (average of 30 measurements

1921 second each) b) Ara h1 on zein-SERS sensor (average of 30 measurements 1 second each) c)

193 background of the zein-SERS sensor (average of 5 measurements 1 second each) d) Ara h1 on

194 zein-SERS sensor (average of 5 measurements 1 second each). Different colors represent

195 individual Raman spectra at mapping points.

197 Furthermore, we could not identify a characteristic marker peak that resulted from the addition of 198 Ara h1 on the zein-SERS platform. Therefore, PCA analysis was applied to the raw data first and 199 the results are shown in Figure 3. In PCA, generally biplots are used in which one axis is one of 200 the principal components and the other axis is another principal component. For most of the 201 cases, PC1 and PC2 is plotted versus each other since those are the two that represent the largest 202 variability in data. However, in certain cases other principal component biplots were used if the 203 variability coming from, the $3^{\text {rd }}$ principal component was more relevant to the analysis being 204 conducted. These biplots help to visualize clusters of data, which have similar variance from the 205 data set, and as a result show similar results to each other. These consistent trends and 206 similarities in the data can be captured that are otherwise very complex and hard to detect. In the 207 context of Raman spectroscopy, PCA biplots are able to differentiate between different samples 208 and/or different concentrations of the sample.

209 The top three plots in Figure 3 below represent PCA analysis for the 30 measurements at 1 210 second acquisition time, whereas the bottom three plots show the 5 measurements at 1 second 211 acquisition time for PC1 vs PC2, PC1 vs PC3 and PC2 vs PC3 from left to right. None of the 212 Principal Component Analysis techniques gave a clear clustering between the background and 213 different concentrations. The reason for this is that varying levels of autofluorescence contribute 214 the largest variation in the data, clouding useful correlations between Raman spectra, and thus 215 the PCA method does not enable differentiation. 

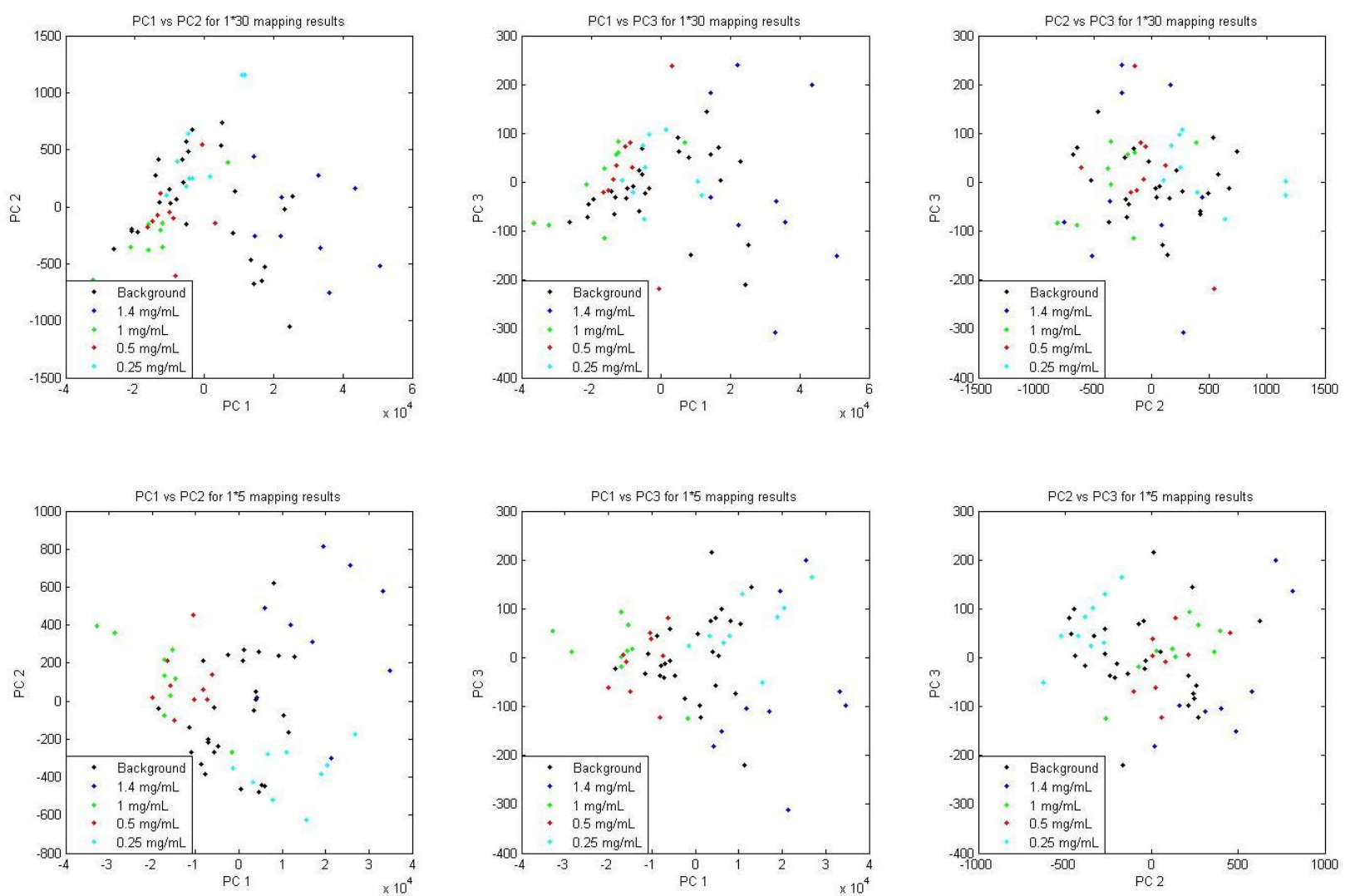

Figure 3. Principal component analysis of Raman spectra for the background and different

218 concentrations of Ara h1. Top-left: PC1 vs PC2, Top-middle: PC1 vs PC3, Top-right: PC2 vs

219 PC3, Bottom-left: PC1 vs PC2, Bottom -middle: PC1 vs PC3, Bottom-right: PC2 vs PC3. PC1 is

220 usually the component that explains the variability best and PC2 is second best with the

221 condition that it is orthogonal to PC1 and PC3 is the third best with the condition that it is

222 orthogonal to both PC1 and PC2.

Analysis of baseline corrected data

Baseline correction was done to eliminate the effect of the broad band and make the

226 baseline linear. Examples of the resulting spectra are shown in Figure 4. Raman spectra in Figure

$2274 a$ and $b$ were baseline subtracted which is the average of 30 measurements at 1 second

228 acquisition time. Figure 4.c and d were collected by using the average of 5 measurements at 1

229 second acquisition time and baseline corrected through polynomial fitting and subtracting. Figure

230 4.a and c shows the background signal coming from the zein-SERS sensor, and Figure 4.b and c

231 represent the spectra when Ara h1 was deposited on the zein-SERS sensor. It was found that 
232 baseline correction was successful in terms of eliminating the variation as a result of auto-

233 fluorescence. As can be seen in Figure 4, spectra at different mapping points (different colors)

234 appear to have the same baseline, intensities and characteristic peaks. It was also found that the

235 Raman spectrum collection was uniform as the mapping points for each of the collection

236 overlapped.
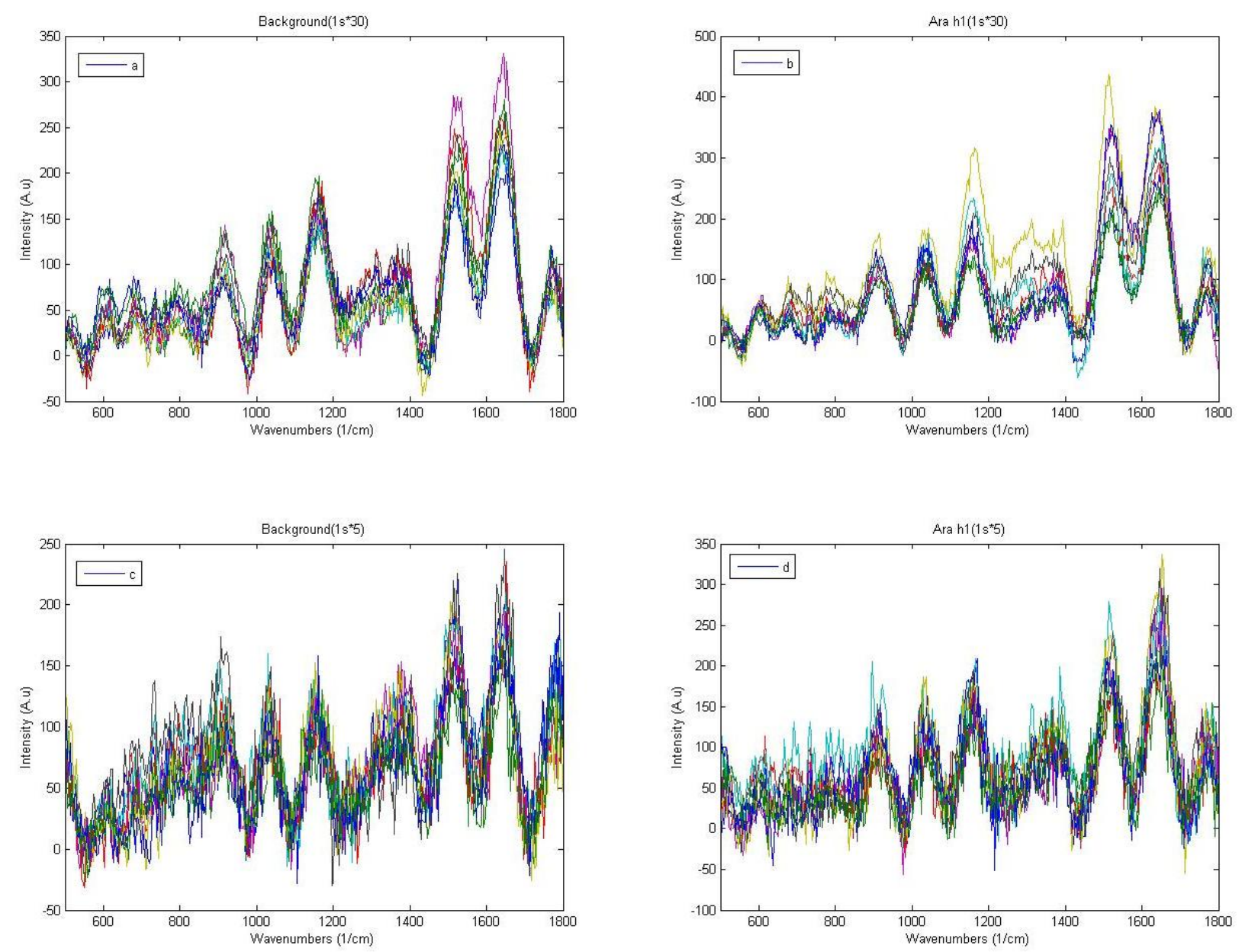

238 Figure 4. Baseline corrected Raman spectra of a) background of the zein-SERS sensor (1s*30

239 acquisition time) b) Ara h1 on zein-SERS sensor (1s*30 acquisition time) c) background of the

240 zein-SERS sensor ( $1 \mathrm{~s} * 5$ acquisition time) d) Ara h1 on zein-SERS sensor ( $1 \mathrm{~s} * 5$ acqusition time)

242 Principal component analysis was applied to the baseline corrected spectra collected both by the

243 average of 30 measurements at 1 second $(1 \mathrm{~s} * 30$ acquisition time and the average of 5

244 measurements at 1 second ( $1 \mathrm{~s} * 5$ acquisition) time. The results for $1 \mathrm{~s} * 30$ acquisition time are

245 shown in Figure 5. It was found that none of the principal component comparisons were able to 
246 differentiate between Ara h1 concentrations and the background. On the other hand, 1s*5

247 acquisition time PCA showed promising results (Figure 6). Both plots of the Raman signals

248 plotted vs. for PC1 and PC2 as well as PC2 and PC3, showed clear differences between the

249 background data and the data with the presence of Ara h1 allergen protein. It was possible to

250 cluster different concentrations of Ara h1 together, which is illustrated in Figure 7.

251

252
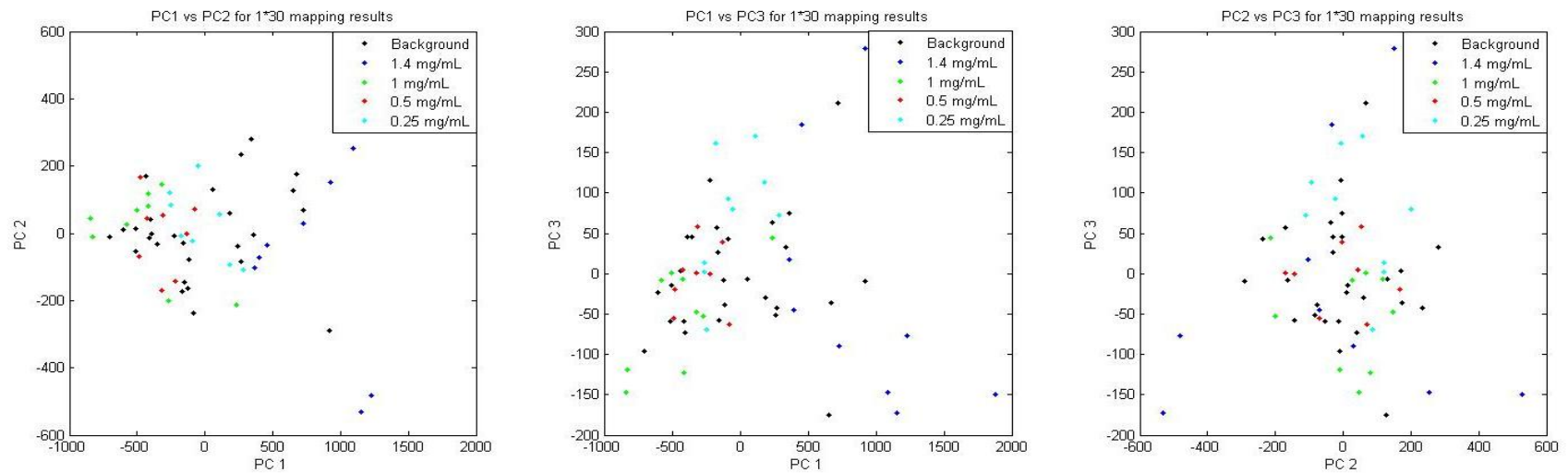

253 Figure 5. Principal component analysis of baseline-corrected Raman spectra for the background 254 and different concentrations of Ara h1 average of 30 measurements at 1 second $(1 \mathrm{~s} * 30$

255 acquisition time. Left: normalized Raman spectral data plotted vs PC1 and PC2, Middle:

256 normalized Raman spectral data plotted vs PC1 and PC3, Right: normalized Raman spectral data 257 plotted vs PC2 and PC3.
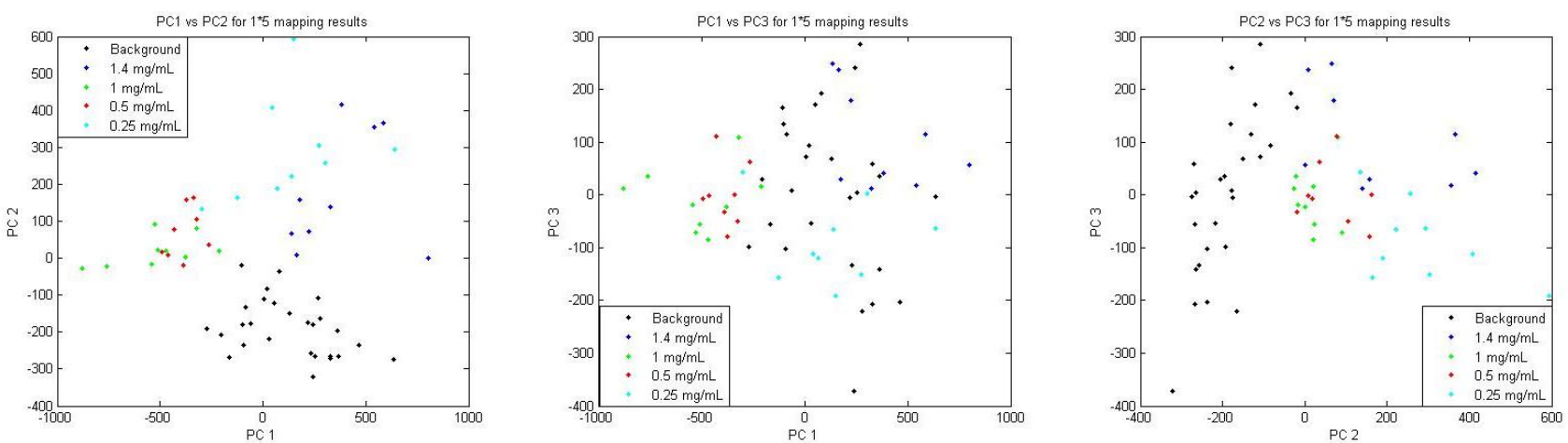

260 Figure 6. Principal component analysis of baseline-corrected normalized Raman spectra for the 261 background and different concentrations of Ara h1 (1s*5 acquisition time). Left: normalized 262 Raman spectral data plotted vs PC1 and PC2, Middle: normalized Raman spectral data plotted vs 263 PC1 and PC3, Right: normalized Raman spectral data plotted vs PC2 and PC3. 

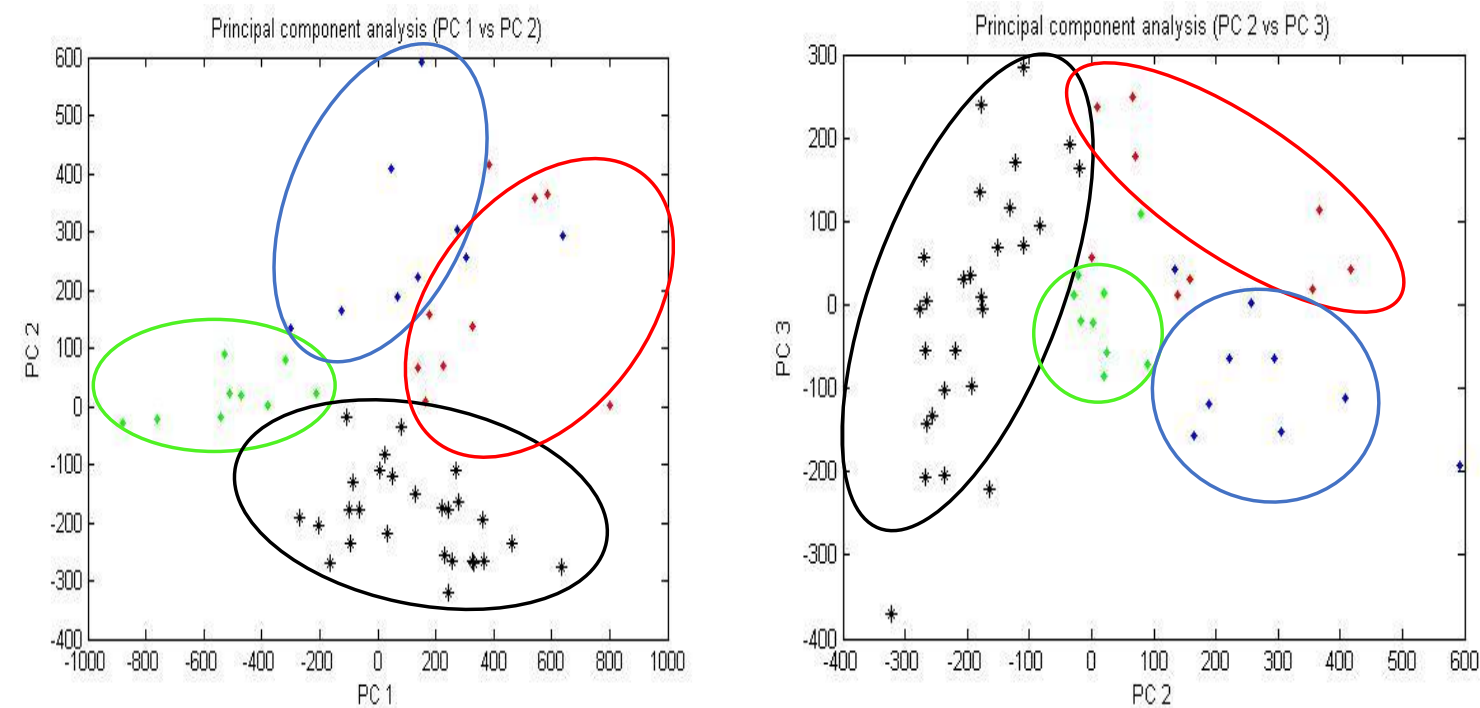

Figure 7. Principal component analysis of baseline-corrected Raman spectra for the background and different concentrations of Ara h1 (1s*5 acquisition time). Left: Normalized Raman spectral intensity data plotted vs the first two principal components PC1 and PC2, Right: Normalized Raman spectral intensity data plotted with respect to principal components PC2 and PC3, Black: background spectral intensity data of zein-SERS platform, Red: Normalized Spectral intensity data for $1.4 \mathrm{mg} / \mathrm{ml}$ Ara h1, Green: Normalized Spectral intensity data for $1 \mathrm{mg} / \mathrm{ml}$, Blue: 0.25 Normalized Spectral intensity data for mg/ml Ara h1.

As shown in Figure 7, when Raman spectral intensity data was plotted vs. PC1 and PC2 as well as PC2 and PC3 it was possible to separate the data into clusters of different concentrations of Ara h1 as well as the background enabling the separation of Raman spectral data of different concentrations. In both normalized Raman spectral data plots vs PC 1 and PC 2 as well as PC 2 and PC 3 plots, background of zein-SERS sensors (black stars) were clustered in a separate region then the Ara h1 protein. It was also observed that different concentrations, $1.4 \mathrm{mg} / \mathrm{ml}$ (red dots), $1 \mathrm{mg} / \mathrm{ml}$ (blue dots), and $0.25 \mathrm{mg} / \mathrm{ml}$ (green dots), were clustered together and formed the elliptic clusters expected from a plot of the data against two principal components. .

284 through baseline correction. Auto-fluorescence makes the biggest contribution to the PCA, which does not leave room for differentiation between samples. Furthermore, when the 
acquisition time was kept longer (1s*30), differentiation was not achieved because auto

287 fluorescence overwhelms Raman signals due to a component of fluorescence which still is not corrected when data acquisition time is longer. However, we found that when the acquisition time is shorter $(1 \mathrm{~s} * 5)$, it was possible to identify the presence and quantify Ara h1 allergen protein on zein-SERS sensors.

Limit of detection (LOD) is considered to be the concentration at or below which it is not

292 possible to detect the presence of the analyte using a sensor. In order to estimate the LOD, serial

293 dilutions were made and we found that the concentration of $0.14 \mathrm{mg} / \mathrm{ml}$ would be the LOD for

294 this zein-SERS sensor. At this concentration, it was not possible to discriminate the background

295 from the case in which Ara h1 was added to the sensor. Figure 8 shows three different principal

296 component analysis plots (PC 1 vs PC 2, PC 1 vs PC 3 and PC 2 vs PC 3). None of these plots

297 were able to form distinguishable clusters, unlike higher concentrations of Ara h1. From this we

298 can conclude that it would not be possible to detect the presence of Ara hl if it's less

299 concentrated than $0.14 \mathrm{mg} / \mathrm{ml}$ in a given sample solution with our zein-SERS sensor. The

300 original concentration of purified protein was $1.4 \mathrm{mg} / \mathrm{ml}$, which was provided in a very minimal

301 amount, after one-log reduction in the concentration, it was not possible to differentiate its

302 presence from the background. However it was possible to differentiate double amount of it. For 303 this reason, we believe it is safe to say that the LOD is $0.14 \mathrm{mg} / \mathrm{ml}$.

304
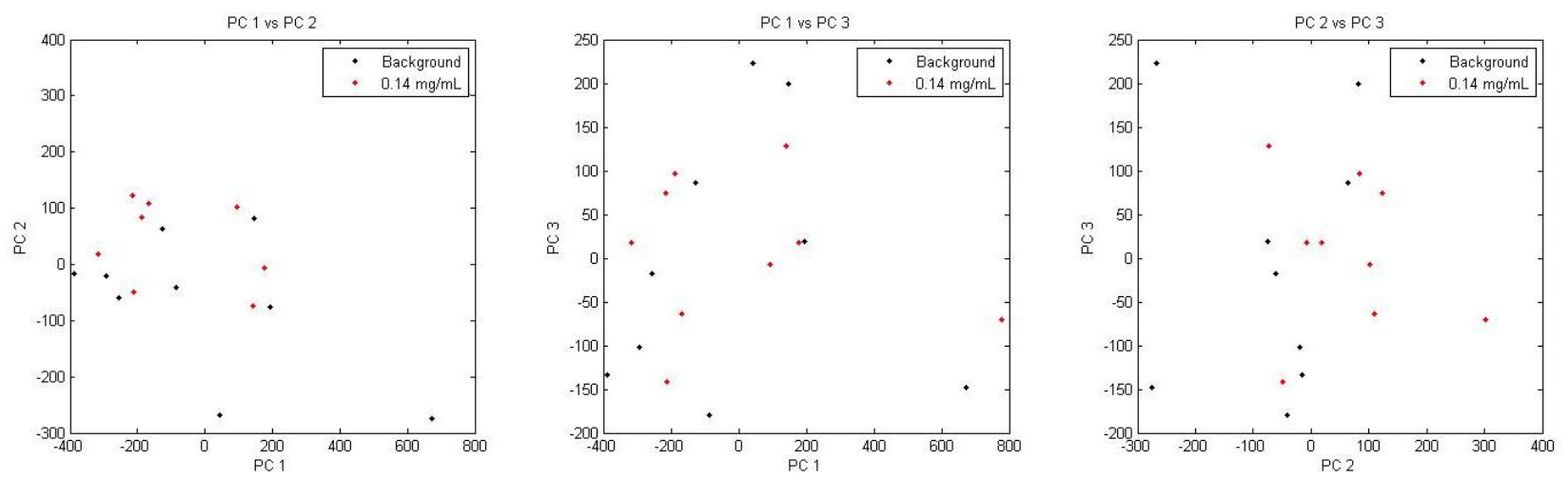

306 Figure 8 Principal component analysis for $0.14 \mathrm{mg} / \mathrm{ml}$ Ara h1 protein on zein-SERS sensor.

307 From left to right: PC1 vs PC2, PC1 vs PC3 and PC2 and PC3.

\subsection{Antibody capturing of Ara h1 on zein-SERS substrates}


In order to functionalize the surface with antibodies, there is a need for a linker molecule.

311 We used 11-MUA as it has both $\mathrm{SH}$ and $\mathrm{CH}_{3}$ ends. $\mathrm{SH}$ molecular group is capable of forming

312 bonds with gold surfaces. Many functionalization protocols require the surface to be immersed in

313 the solution containing the target molecule, however for the zein-SERS platform, this posed a

314 problem of disintegration because zein is soluble in $70 \%$ ethanol. Figure 9 shows the

315 disintegration of the zein-SERS sensor after being immersed in ethanol solution of 11-MUA.

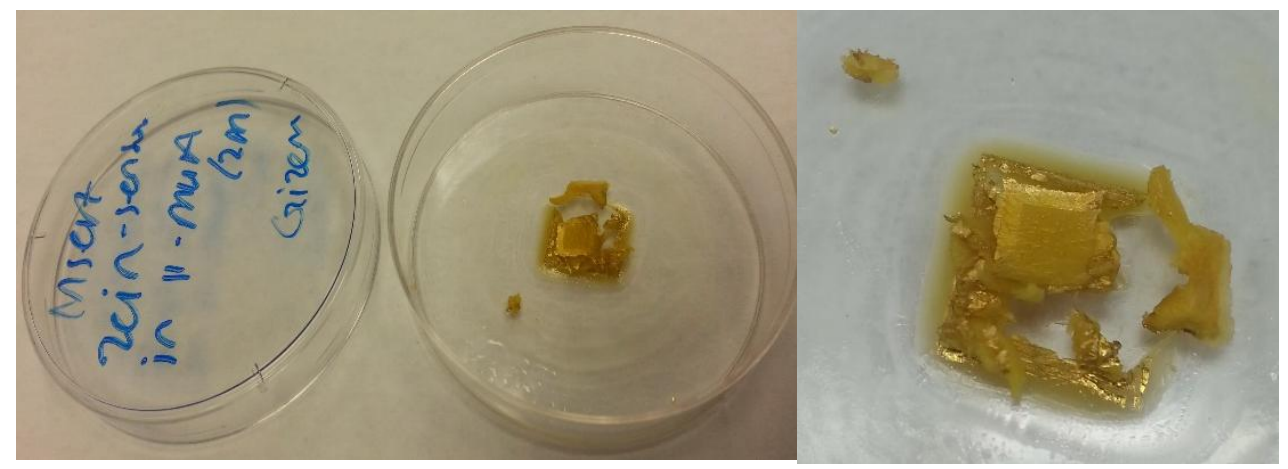

317 Figure 9. Left: Zein-SERS platform, after being immersed in 2M of 11-MUA solution inside a 318 petri dish. Right: Close-up image of the zein-SERS sensor.

In order to avoid disintegration, a droplet of 2M 11-MUA was dropped on the area covering only the pyramid-patterned area was used to functionalize the surface. This also created some distortion on the sensor, due to the absorbance of the solvents. In order to account for the effect of the solvents being absorbed by the sensor, a control consisting of the original gold zein

324 film was used. All functionalization steps were applied to the control, except for the active materials (11-MUA, EDC-NHS solution, 2F7 antibody and Ara h1). For instance, the same volume of ethanol was used instead of the 11-MUA in ethanol solution or same volume of water was used instead of the aqueous solution of antibody. The control sensor was also exposed to all the washing and drying steps that occurred during the process of functionalization. For simplicity, in this section, this control sensor will be referred to as background zein-SERS sensor, as it represents the potential Raman signals coming from the sensor + side effects of the functionalization process.

Furthermore, a gold surface (coated on PET) and gold coated pyramid patterns on PET were also used as a control to ensure the functionalization and Ara h1 capturing was successful.

334 Figure 10 shows the baseline subtracted and averaged Raman signatures of Ara h1 on 335 functionalized surfaces after rinsing process: smooth gold surface, PET-SERS substrate, and 
zein-SERS substrate. As expected, the smooth gold surface gave the lowest intensity signal, since there is no SERS effect in the absence of nanopatterns. PET-SERS substrate showed enhancement in the Raman signal of the protein peaks, whereas zein-SERS showed the strongest signal. This enhancement observed with the zein-SERS surface might have been caused by a cumulative effect of the background plus Ara h1 protein.

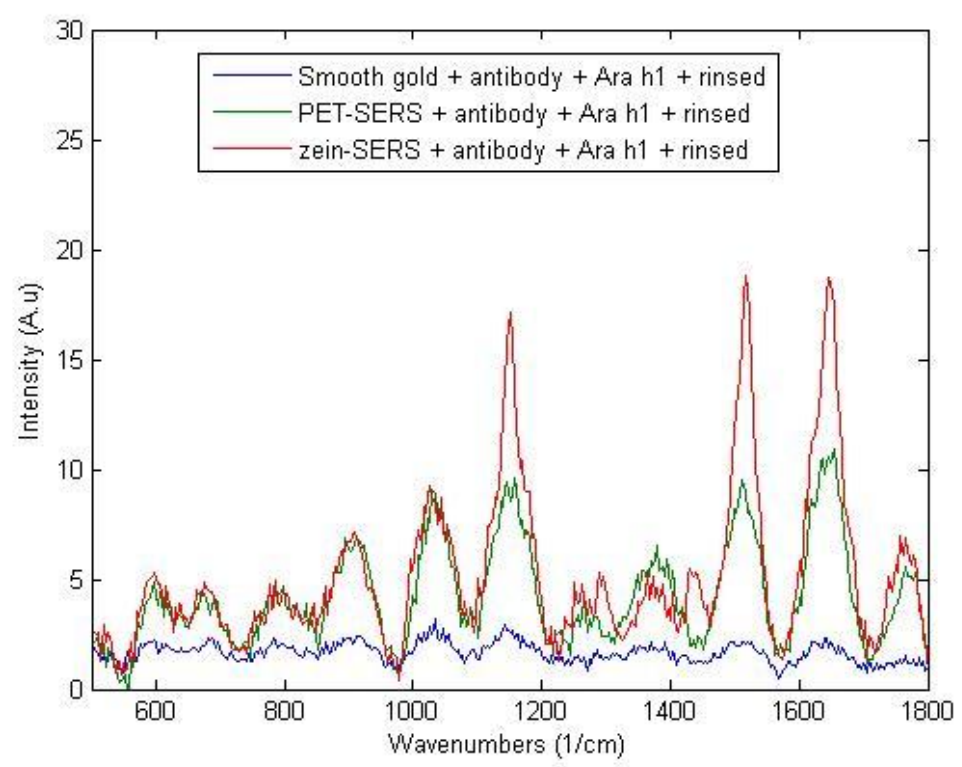

Figure 10. Raman spectra generated by Ara h1 protein captured with $2 \mathrm{~F} 7$ antibody on different platforms. Red: zein-SERS, green: PET-SERS, blue: smooth gold.

In order to discriminate between the background, antibody functionalization and captured Ara h1, PCA analysis was conducted for Raman spectra collected on zein-SERS platform as well as smooth-gold and PET-gold-pyramid surface. Plots of PC1 vs PC2 and PC1 and PC3 are shown in Figure 11. The color representations are as follows: black: baseline-corrected Raman spectra for background zein-SERS sensor, pink: antibody-functionalized zein-SERS sensor, red: Ara h1 protein captured by antibody-functionalized zein-SERS sensor, cyan: Ara h1 protein on functionalized zein-SERS sensor-not rinsed, blue: Ara h1 protein captured by antibodyfunctionalized smooth gold surface, and green: Ara h1 protein captured by antibodyfunctionalized PET-SERS substrate. It was observed that principal component 1 was successful in differentiating different platforms from each other, namely smooth-gold (blue), PET-SERS (green) and zein-SERS (all the other colors), however it was not successful in differentiating the presence/ absence of Ara h1 on zein-SERS. It was concluded that the first principal component 
was representative of different substrates in this case and was not reliable in discriminating Ara

358 h1 protein captured by the antibody.

Plots of normalized Raman intensity scores vs PC2 and PC3 gave successful results in clustering these three cases in separate groups (Figure 12). Antibody functionalized zein-SERS sensor formed a separate cluster (blue circle) compared to the background (green circle), which indicated the ability of PCA to discriminate successfully the functionalization of the antibody on

363 the surface. In addition, Ara h1 captured with these antibodies also ended up in a different cluster 364 (red). This shows that it is possible to differentiate the presence of Ara h1 protein that is captured by 2 F7 monoclonal antibody which was immobilized onto the surface of zein-SERS substrate. To further validate the correlation of the Ara h1 cluster (red) with the presence of protein, a comparison made by adding the Raman spectra of the non-zein substrates into the PCA analysis. Figure 13 shows the plot for PC 2 vs PC 3 for 5 different cases: Ara h1 on smooth gold surface (cyan), Ara h1 on PET-SERS substrate (green), Ara h1 on zein-SERS substrate (red), antibodyfunctionalized zein-SERS substrate (blue) and background of zein-SERS sensor (black).

371 Whenever Ara h1 protein was present on a given substrate, it fell in the red cluster. This 372 indicates that analysis of the Raman spectra by plotting normalized scores vs. PC 2 and PC 3 is a 373 successful method to detect the presence of Ara h1 for our given system.
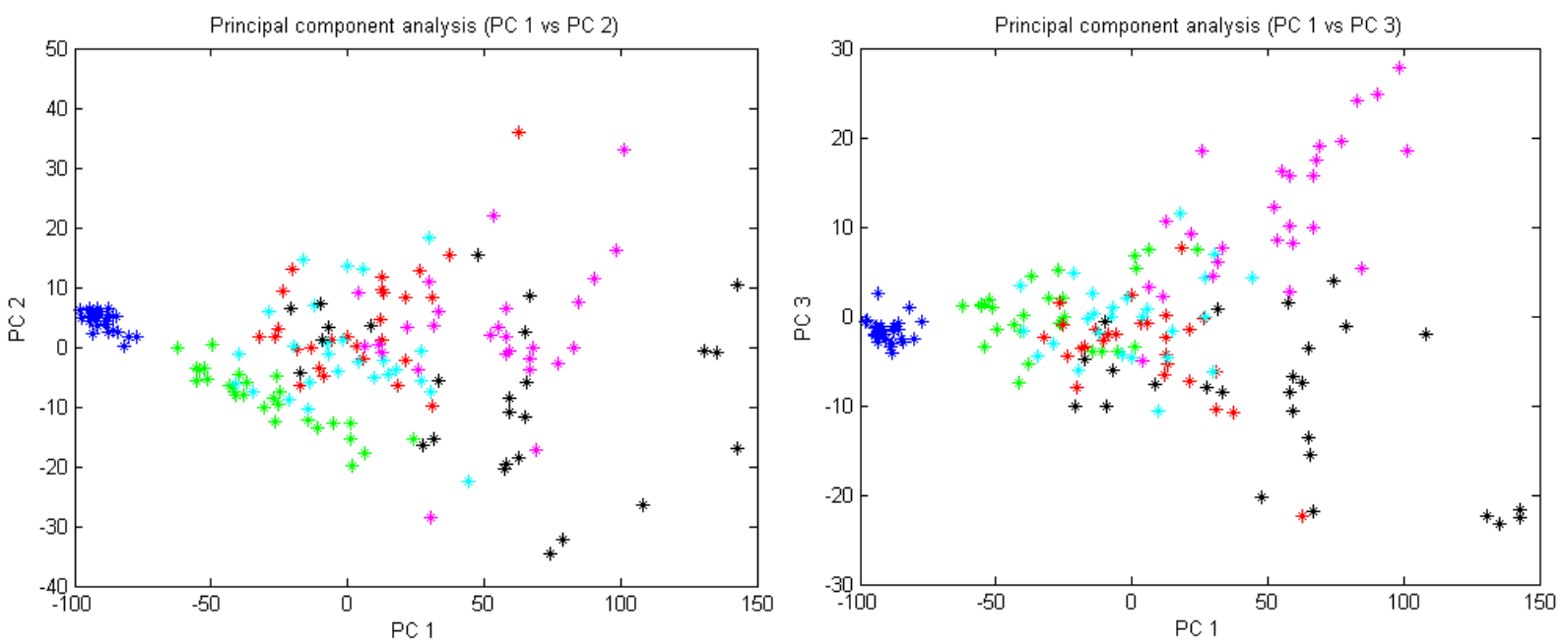

Figure 11. Principal component analysis PC1 vs PC2 (Left) and PC1 vs PC3 (Right) of

377 baseline-corrected Raman spectra for background zein-SERS sensor (black), antibody-

378 functionalized zein-SERS sensor (pink), Ara h1 protein captured by antibody-functionalized

379 zein-SERS sensor (red), Ara h1 protein on functionalized zein-SERS sensor-not rinsed(cyan), 

protein captured by antibody-functionalized PET-SERS substrate (green)

382

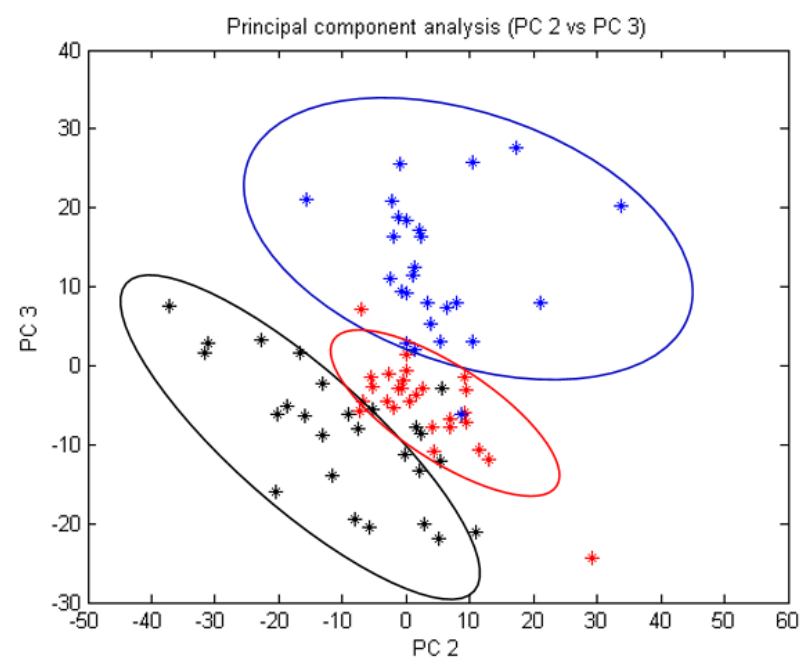

383

384

385 386

Figure 12. Principal component analysis (PC2 vs PC3) of baseline-corrected Raman spectra for background zein-SERS sensor (black), antibody-functionalized zein-SERS sensor (blue) and Ara h1 protein captured by antibody-functionalized zein-SERS sensor (red)

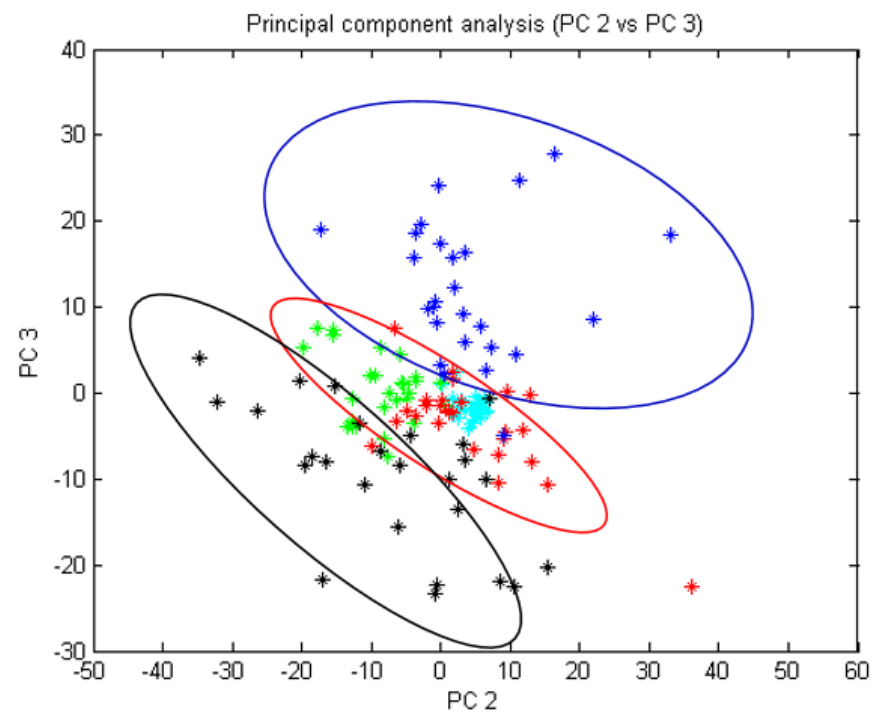

Figure 13. Principal component analysis (PC2 vs PC3) of baseline-corrected Raman spectra for background zein-SERS sensor (black), antibody-functionalized zein-SERS sensor (blue) and Ara h1 protein captured by antibody-functionalized zein-SERS sensor (red), Ara h1 protein 
captured by antibody-functionalized smooth gold surface (cyan), Ara h1 protein captured by

392 antibody-functionalized PET-SERS substrate (green)

393

394

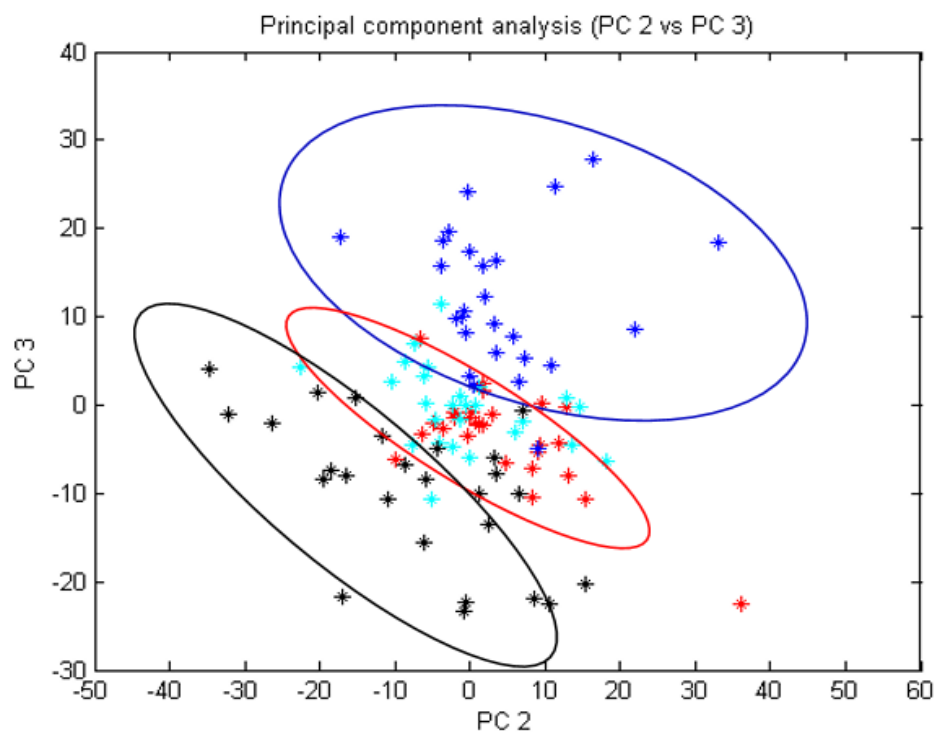

395

396

397

398

399

400

401

402

403

404

405

406

407

408

409

410
Figure 14. Principal component analysis (PC2 vs PC3) of baseline-corrected Raman spectra for background zein-SERS sensor (black), antibody-functionalized zein-SERS sensor (blue) and Ara h1 protein captured by antibody-functionalized zein-SERS sensor (red), Ara h1 protein on functionalized zein-SERS sensor- not rinsed (cyan)

A rinsing process was applied to remove the excess Ara h1 that was not bound to the monoclonal antibody on the surface. An experiment was carried out to compare the before and after rinsing of Ara h1 on the functionalized surface. PCA analysis (Figure 14) showed that before (cyan color) and after (red color) rinsing fell inside the same cluster, with a slight shift within the cluster after the rinsing process. This result proved that the red cluster is consistently correlated with Ara h1 protein. In addition, 2F7 monoclonal antibody was successful in bonding to the protein as evident by the signal after the rinsing process. 
This research have shown that a biodegradable sensor platform made out of zein was capable

412 of detecting peanut allergen protein with the surface enhanced Raman spectroscopy technique

413 combined with principal component analysis (PCA). Detection and quantification gave

414 promising results when Raman spectra were treated with baseline corrections. On the other hand,

415 autofluorescence resulted in data that were not distinguishable from the background. Since zein

416 is a biological molecule, it has inherent autofluorescence, which can hinder data analysis at

417 times. However, post-processing of the data combined with statistical clustering technique gave

418 results in which quantification was possible.

419 Another challenge observed while manufacturing and utilizing zein biopolymer in a sensor

420 platform was the deformation or distortion as a result of solvent treatment during

421 functionalization process. This obstacle was overcome by applying minimal volume on the gold

422 surface of the sensor, that helped protect the integrity of the sensor. Furthermore, PCA results not

423 only showed that the functionalization process was successful, but also it was possible to detect

424 the LOD by capturing with an antibody, providing the specificity of the sensor.

\section{Conclusion}

In this research, detecting peanut allergen protein, Ara h1 on a biodegradable gold/zein film

428 SERS platform was developed for the first time. It was possible to detect and quantify Ara h1

429 protein by using principal component analysis (PCA). Even though the background of the sensor

430 overlapped with the peaks obtained from Ara h1, the PCA method was found to be successful to

431 differentiate Ara h1 with varying concentrations to separate clusters. This was only achieved

432 when the baseline corrected Raman spectra was used; raw spectra did not give enough

433 differentiation through PCA analysis. Furthermore, specificity of the sensor was obtained

434 through functionalization of the gold surface with monoclonal antibody of Ara h1. PCA analysis

435 enabled distinction of Ara h1 protein captured by antibodies from background of the sensor, as

436 well as only antibody-functionalized surface. This was a proof-of-concept that zein-SERS

437 sensors can be used in detecting allergen protein, Ara h1 by capturing it with monoclonal

438 antibodies. Further studies are needed to improve sensitivity, as well as application to real food

439 systems. 


\section{Acknowledgments}

442 This study was funded by USDA hatch funds. The authors declare that there was no conflict of 443 interest.

\section{References}

447 [1] M. Osterballe, T. K. Hansen, C. G. Mortz, A. Høst, and C. Bindslev-Jensen, The prevalence 448 of food hypersensitivity in an unselected population of children and adults. Pediatric Allergy and 449 Immunology, 16 (2005) 567-573.

[2] Z. Xiaoyan, L. Ruiyi, L. Zaijun, L. Junkang, G. Zhiguo, \& W. Guangli, A surface-enhanced 452 Raman scattering strategy for detection of peanut allergen Ara h 1 using a bipyramid-shaped 453 gold nanocrystal substrate with an improved synthesis. RSC Advances, 4:30 (2014) 1536345415370.

455 [3] A. Pomés, R. M. Helm, G. A. Bannon, A. W. Burks, A. Tsay, \& M. D. Chapman, 456 Monitoring peanut allergen in food products by measuring Ara h 1, Journal of Allergy and 457 Clinical Immunology, 111:3 (2003) 640-645.

[4] S.J. Koppelman, R. A. A. Vlooswijk, L. M. J. Knippels, M. Hessing, E. F. Knol, F. C. Van

460 Reijsen, \& C. A. F. M. Bruijnzeel-Koomen, Quantification of major peanut allergens Ara h 1 and 461 Ara h 2 in the peanut varieties Runner, Spanish, Virginia, and Valencia, bred in different parts of 462 the world. Allergy, 56:2 (2001) 132-137.

464 [5] W. Burks, H. A. Sampson \& G. A. Bannon, Peanut allergens. Allergy, 53 (1998) 725-730.

466 [6] H. W. Wen, W. Borejsza-Wysocki, T. R. DeCory, \& R. A. Durst, Development of a 467 competitive liposome-based lateral flow assay for the rapid detection of the allergenic peanut 468 protein Ara h1, Analytical and bioanalytical chemistry, 382:5 (2005) 1217-1226. 
470 [7] J. Zheng, S. Pang, T. P. Labuza, \& L. He, Evaluation of surface-enhanced Raman scattering 471 detection using a handheld and a bench-top Raman spectrometer: A comparative study, Talanta, $472129(2014) 79-85$.

473

474 [8] T. Lang, S. Pang, \& L. He, Integration of colorimetric and SERS detection for rapid

475 screening and validation of melamine in milk, Analytical Methods, 7:15)(2015) 6426-6431.

476

477 [9] K. Weidemaier, E. Carruthers, A. Curry, M. Kuroda, E. Fallows, J. Thomas, D. Sherman \&

478 M. Muldoon, Real-time pathogen monitoring during enrichment: a novel nanotechnology-based

479 approach to food safety testing, International journal of food microbiology, 198 (2015). 19-27.

481 [10] P.G. Gezer, A. Hsiao, J.L. Kokini, G. L. Liu, Simultaneous transfer of noble metals and 482 three-dimensional micro and nano-patterns onto zein for fabrication of nanophotonic platforms, 483 Journal of Material Science (2015) (under review)

485 [11] J. Zheng, S. Pang, T.P. Labuza \& L. He, Semi-quantification of surface-enhanced raman 486 scattering using a handheld raman spectrometer: a feasibility study. Analyst, 138: 23 (2013). $487 \quad 7075-7078$.

489 [12] C. V. Di Anibal, L. F. Marsal, M. P. Callao, \& I. Ruis'anchez, Surface enhanced raman 490 spectroscopy (sers) and multivariate analysis as a screening tool for detecting sudan i dye in 491 culinary spices. Spectrochimica Acta Part A: Molecular and Biomolecular Spectroscopy, 87 492 (2012) 135-141.

493

494 
Graphical Abstract
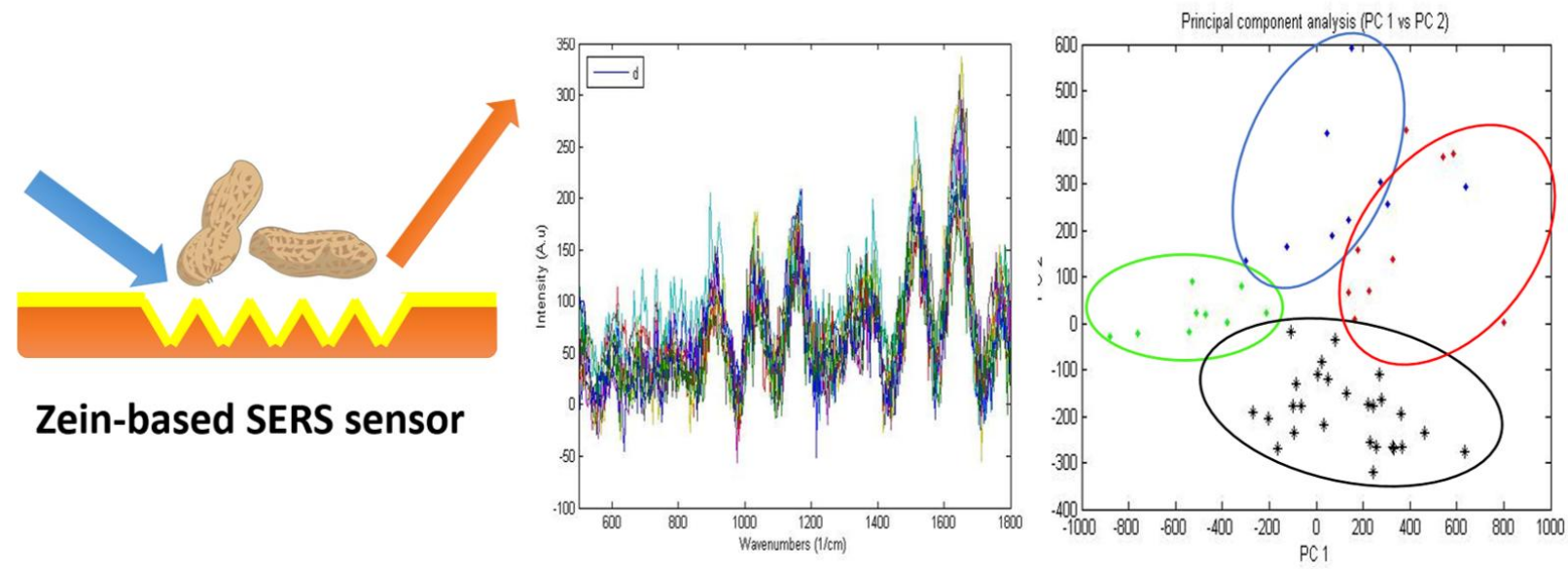